PROCEEDINGS OF THE

AMERICAN MATHEMATICAL SOCIETY

Volume 128, Number 2, Pages 621-622

$\mathrm{S}$ 0002-9939(99)05175-8

Article electronically published on July 27,1999

\title{
BLASCHKE PRODUCTS AND EXPANDING MAPS OF THE CIRCLE
}

\author{
DAVID TISCHLER
}

(Communicated by Jozef Dodziuk)

\begin{abstract}
For an analytic map of the closed unit disk onto itself that leaves the boundary circle invariant, we give necessary and sufficient conditions for the map of the boundary to be expanding.
\end{abstract}

Let $B$ be an analytic map from the closed unit disk onto itself that maps the boundary circle into itself. Suppose $B$ is expanding when restricted to the boundary. In this case, it is known that there is a fixed point in the interior of the disk. However the converse is not true. In this note we will give a couple of necessary and sufficient conditions for $B$ to be expanding on the boundary circle, one of which gives an estimate on the derivative of the expanding map. A map $B$ as above is a constant multiple of a finite Blaschke product.

Let $D_{r}=\{z \in \mathbf{C}:|z|<r\}$ and $C_{r}=\{z \in \mathbf{C}:|z|=r\}$. Let $a=\left\{a_{1}, \ldots, a_{n}\right\}$. The finite Blaschke product $B_{a}$ is defined by the formula $B_{a}(z)=\prod_{i=1}^{n} \frac{z-a_{i}}{1-\bar{a}_{i} z}$, where $B_{a}\left(D_{1}\right)=D_{1}$ and $B_{a}\left(C_{1}\right)=C_{1}$. Let $\tau_{a}$ be the restriction of $B_{a}$ to $C_{1}$. We will give conditions on $B_{a}$ so that $\tau_{a}$ is expanding and an estimate for $\left|\tau_{a}^{\prime}\right|$. Compare with Martin [2], where a sufficient condition for $\tau_{a}$ expanding is given.

Theorem 1. The following three conditions are equivalent.

i) $\left|\tau_{a}^{\prime}\right|>1$ for $z \in C_{1}$.

ii) For some $r_{1}<1, B_{a}\left(D_{r_{1}}\right)$ is contained in the interior of $D_{r_{1}}$.

iii) For all $\lambda \in C_{1}, \lambda \cdot B_{a}$ has a fixed point in the interior of $D_{1}$.

Theorem 2. i) Let $M_{r}=\max \left|B_{a}(z)\right|$ for $z \in C_{r}$. Suppose $M_{r_{1}}<r_{1}$ is as in condition ii) of Theorem 1. Let $k=\log M_{r_{1}} / \log r_{1}$. Then $\left|\tau_{a}^{\prime}(z)\right| \geq k>1$ for $z \in C_{1}$.

ii) Let $A=\left\{r: B_{a}\left(D_{r}\right)\right.$ is contained in the interior of $\left.D_{r}\right\}$. Then $A$ is an interval with 1 as an endpoint.

Proof of Theorem 1. i) $\Rightarrow$ ii). For $z \in C_{1}, B_{a}^{\prime}(z)=\tau_{a}^{\prime}(z)$. Therefore, the derivative of $B_{a}$ normal to $C_{1}$ is greater than 1 . Since $C_{1}$ is invariant by $B_{a}$, for $r$ sufficiently close to $1, B_{a}\left(D_{r}\right) \subset$ interior of $D_{r}$.

ii) $\Rightarrow$ iii). This follows from the Brouwer fixed point theorem and the fact that $\lambda \cdot B_{a}$ satisfies ii) whenever $B_{a}$ does.

iii) $\Rightarrow$ i). $B_{a}$ has $n+1$ fixed points on the Riemann sphere. Note that $B\left(\frac{1}{z}\right) \overline{B(z)}=$ 1 for all $z \in \mathbf{C}$. If $B_{a}$ has a fixed point in the interior of $D_{1}$, then it has one in

Received by the editors April 14, 1998.

1991 Mathematics Subject Classification. Primary 58F03, 30D50.

(C)1999 American Mathematical Society 
the exterior of $D_{1}$. Hence there are at most $n-1$ fixed points on $C_{1}$. Since $\tau_{a}$ has topological degree $n, B_{a}$ must have at least $n-1$ and hence exactly $n-1$ fixed points on $C_{1}$. The same is true for $\lambda \cdot B_{a}$ for $\lambda \in C_{1}$. Let $\tilde{\tau}_{a}: R \Rightarrow R$ be a lift of $\tau_{a}$ to the universal covers. Then $\tilde{\tau}_{a}(u)-u+$ constant restricted to a fundamental domain of the covering must intersect $n-1$ different lifts of $1 \in C_{1}$. This implies $\tilde{\tau}_{a}(u)-u+$ constant must have a positive derivative. If the derivative were zero at some point, then $\tilde{\tau}_{a}(u)-u+$ constant $=0$ would have a multiple root for some constant and hence, for some $\lambda \in C_{1}, \lambda \cdot B_{a}$ would have more than $n-1$ fixed points in $C_{1}$. If $\tilde{\tau}_{a}(u)-u$ has a positive derivative, then $\left|\tilde{\tau}^{\prime}(u)\right|>1$ for all $u$ and hence $\left|\tau_{a}^{\prime}\right|>1$ for all $z \in C_{1}$.

Proof of Theorem 2. Let $r_{1} \leq r \leq r_{2}=1$. Hadamard's Three Circle theorem asserts that $M(r) \leq M\left(r_{1}\right)^{a} M\left(r_{2}\right)^{1-a}$, where $a=\log \left(r_{2} / r\right) / \log \left(r_{2} / r_{1}\right)$; see Alhfor's [1]. Suppose $M_{r_{1}}<r_{1}$. Let $k=\log \left(M_{r_{1}}\right) / \log \left(r_{1}\right)>1$. Let $r_{2}=1$ in Hadamard's inequality. Since $M_{r_{2}}=1$ we obtain $M_{r} \leq r^{k}$ for $r_{1} \leq r \leq 1$. Note that $M_{1}=1$ and $\left(r^{k}\right)^{\prime}=k$ when $r=1$. Therefore the derivative of $B_{a}$ normal to $C_{1}$ is greater than or equal to $k$. Therefore $\left|B_{a}^{\prime}(z)\right| \geq k$ for $z \in C_{1}$ and hence $\left|\tau_{a}^{\prime}(z)\right| \geq k$. This proves part i). Since $r^{k}<r$ for $r<1$ we see that $M_{r}<r$. This shows that $A$ is an interval which completes the proof.

Remark 1. Theorem 2. i) shows that in Theorem 1, ii) $\Rightarrow$ i).

Remark 2. In Theorem 1, condition iii) is equivalent to a certain discriminant being negative for all $\lambda \in C_{1}$. However, it does not appear any easier to verify the discriminant condition than verifying $\left|\tau_{a}^{\prime}\right|>1$ directly. The discriminant is obtained as follows. There is a linear fractional transformation of $\mathbf{C}$ that takes the real line onto $C_{1}$. Conjugating $\lambda \cdot B_{a}$ by this map gives a rational function $f / g$ with real coefficients. This function has either exactly one pair of complex conjugate fixed points or none, depending on whether or not condition iii) is satisfied. The discriminant of $f(z)-z g(z)$ is negative if and only if there is one pair of complex conjugate fixed points for $f / g$.

\section{REFERENCES}

1. L. Ahlfors, Complex Analysis, McGraw-Hill, New York, 1966. MR 32:5844

2. N. F. G. Martin, On finite Blaschke products whose restrictions to the unit circle are exact endomorphisms, Bull. London Math. Soc. (15) 1983 (4), pp. 343-348. MR 84h:30045

Department of Mathematics, Queens College (CUNY), Flushing, New York 113671597 